\title{
La gestión de costos basada en actividades como herramienta estratégica para la toma de decisiones en las empresas constructoras de la ciudad de Loja, Ecuador
}

\section{Activity-Based Costing (ABC) System a Strategic Tool for Decision-Making \\ Within Construction Companies in the city of Loja, Ecuador}

Paola de los Ángeles Escandón Guillén. ${ }^{1}$, Cecilia Ivonne Narváez Zurita. ${ }^{2}$ Juan Carlos Eràzo Álvarez. ${ }^{3}, \&$ Jorge Edwin Ormaza Andrade. ${ }^{4}$

DOI: https://doi.org/10.33262/visionariodigital.v3i2.1.581

\begin{abstract}
.
Determining the cost of production is a fundamental tool for construction companies and its effective performance in the competitive market. Therefore they must have a budgeting and costs control model to achieve the integration of all the circuits operation and thus enough information to the wise decision-making.

Consequently, the objective of this article is to verify that Activity-Based Costing (ABC) System has become a strategic tool to enhance the business process management in the construction business in the city of Loja, Ecuador. This study combines features of the applied research which were developed using a mixed approach and qualitative method.

The main techniques used were surveys and interviews to all executives and staff of the Company OSMAR Constructions. The results identified that there is a lack of costs

1 Universidad Católica de Cuenca, Posgradista Maestría en Contabilidad y Auditoría, Cuenca, Ecuador, pdescandong951@psg.ucacue.edu.ec

2 Universidad Católica de Cuenca, Posgrados, Cuenca, Ecuador. inarvaez@ucacue.edu.ec

${ }^{3}$ Universidad Católica de Cuenca, Posgrados, Cuenca, Ecuador. jcerazo@ucacue.edu.ec

${ }^{4}$ Universidad Católica de Cuenca, Posgrados, Cuenca, Ecuador, jormaza@ucacue.edu.ec
\end{abstract}


production determination; therefore, $\mathrm{ABC} / \mathrm{ABM}$ is an accurate tool to control and manage these kinds of industries.

Keywords: Activity-Based Costing $\mathrm{ABC} / \mathrm{AB}$ System, activities, indirect costs, Decision Making.

\section{Resumen}

La determinación de los costos de producción constituye una herramienta primordial para que las empresas constructoras puedan tener un efectivo desempeño en el actual mercado competitivo, para ello, es imprescindible que cuenten con modelos de gestión de costos y presupuestos que logren la integración de todo el circuito de operación a fin de generar la suficiente información para la acertada toma de decisiones.

En tal razón, el objetivo de este artículo es demostrar como el costeo basado en actividades se convierte en una herramienta estratégica para mejorar la gestión de los procesos empresariales de la industria de la construcción en la ciudad de Loja, Ecuador. El presente estudio reúne las características de una investigación aplicada, la cual se desarrolló bajo un enfoque mixto, siento el método cualitativo el de mayor prevalencia. Las principales técnicas que se utilizaron en la investigación fueron la encuesta y la entrevista las mismas que se aplicaron a los directivos y personal de la empresa OSMAR Construcciones, los resultados obtenidos permitieron identificar las principales falencias en cuanto a la determinación de los costos de producción y por ende confirman que el sistema de costos $\mathrm{ABC} / \mathrm{ABM}$ se constituye en una herramienta de control y gestión apropiada para este tipo de industrias.

Palabras clave: gestión de costos $\mathrm{ABC} / \mathrm{ABM}$, actividades, costos indirectos, toma de decisiones. 


\section{Introducción}

Hoy en día, existe la creencia de que la contabilidad de gestión basada en actividades, es un elemento indispensable, estratégico y funcional que se aplica únicamente en las grandes empresas; dicho pensamiento es totalmente erróneo, en razón, que ésta contabilidad al ser establecida dentro de las pequeñas y medianas empresas proporciona resultados positivos y grandes beneficios para estas organizaciones; una muestra o ejemplo de su aplicación se evidencia mediante: la accesibilidad a toda la información de la empresa referente al stock, control de la gestión y cálculo de costeo de cada una de las áreas de producción, convirtiéndose en una herramienta de gestión estratégica que la gerencia debe usarla para tomar decisiones (Calvo, 2017).

La contabilidad de gestión es una de las mejores herramientas que pueden tener las empresas con procesos de producción debido a que sus elementos tienen una mayor complejidad al momento de determinar de forma correcta el costo de un producto, la aplicación de este tipo de contabilidad permitirá calcular de forma correcta el costo real del bien sobre el que se esté trabajando y con ello lanzarlo al mercado de manera más efectiva, haciendo uso de las estrategias necesarias que permitan obtener los mejores resultados. Algunos de los cálculos no son fáciles, sin embargo, es responsabilidad de cada empresa adaptarlo de la manera que considere mejor.

Estupiñan (2006) señala que la contabilidad de gestión puede definirse como la caracterización, medida, acumulación, investigación, elaboración, interpretación y notificación de los datos obtenidos, que usan los altos directivos para planear, retroalimentar y vigilar dentro de la institución, así como para afirmar la utilización de los recursos y la responsabilidad sobre ellos. Los autores Valdez, Nazareno y Toala (2017) mencionan que con el propósito de apoyar a la toma de decisiones gerenciales e incrementar el nivel de eficiencia y eficacia en la administración es imprescindible la aplicación de las técnicas y conceptos contables para procesar los datos económicos, históricos y estimados de una organización, todo esto con el establecimiento de planes basados en objetivos económicos racionales. El contenido en este tipo de contabilidad se fija por las exigencias informativas que exige el control directivo y se presenta de forma analítica, por centros de producción, líneas de negocio o líneas de producto. 
Al respecto, Chezpik (2007) menciona que la contabilidad de gestión se encarga de percibir, medir y cotizar la circulación interna de la compañia, racionalizando y controlando los recursos propios para abastecer a los directivos de información indispensable y suficicente que logre acatar decisiones en el ámbito interno y a corto plazo en función de la organización, conforme a los fines propuestos. Di igual manera, Amat y Soldevila (2011) manifiestan que la contabilidad de gestión aporta información económica para posibilitar a los usuarios de la misma, el diagnóstico del negocio y la toma de decisiones. La contabilidad de gestión pretende, teniendo en cuenta el contexto del cambio continuo, dar respuestas a las debilidades presentadas en el seno de las organizaciones. La aplicación de este tipo de contabilidad en cualquier empresa nace de la necesidad imperiosa de ser competitiva.

Por otra parte, Lawrence (2002) indica que la contabilidad de costos es un proceso ordenado que usa los principios generales de contabilidad para registrar los costos de operación de un negocio de tal manera que, con datos de producción y ventas, la gerencia pueda usar las cuentas para averiguar los costos de producción y los costos de distribución, ambos por unidad y en total de uno o de todos los productos fabricados o servicios prestados, y los costros de otras funciones diversas de la negociación, con el fin de lograr una operación económica, eficiente y productiva.

Asimismo, (Molina, 2002) afirma que inmersa en la contabilidad general o financiera como una etapa ampliada está la contabilidad de costos de una entidad manufacturera que suministra aceleradamente al nivel gerencial los datos relativos a los costos de producción o ventas. El costo se define como toda salida o gasto de dinero o su equivalente para lograr algun bien o servicio, mediante la disminución de activos o al incidir en pasivos en el momento en que se consiguen los beneficios.

Los autores antes citados señalan por separado definiciones, posiciones y conceptualizaciones de contabilidad de gestión y de costos; ahora bien, el autor (Lopéz, 2011) por su parte, define a la contabilidad de gestión o de costos, como un segmento especializado de la contabilidad general con un ambiente de aplicación dentro de las empresas industriales cuya importancia radica en:

- Controlar el costo de producción en cada una de las fases de elaboración del producto. 
- Realizar un uso adecuado de los materiales de producción.

- Determinar el costo de los productos en proceso, productos terminados y el margen de utilidad de los productos terminados, $\mathrm{y}$.

- Proveer herramientas útiles de planificación y control de los costos de producción que faciliten la toma de decisiones a la gerencia.

\section{Sistemas de costos ABC}

Los costos $\mathrm{ABC}$ priorizan los procesos y no los costos, ya que el objetivo de este sistema de costeo es conocer y distribuir correctamente las actividades Lopéz (2011). Autores como (Kaplan y Cooper , 2006) indican que el sistema de costo basado en actividades logra que los costos indirectos vayan primero a las actividades y procesos para luego dirigirse a los productos, servicios y clientes. Por lo que este sistema contribuye con información mas clara sobre los costos de operaciones y su valor real. (Zapata, 2007) al respecto afirma que, la aplicación de costos ABC facilita a las empresas el manejo de su estructura de costos totales, manteniendo una correcta distribucion de ellos y relacionando las actividades con los costos, permitiendo de esta forma un correcto sistema de gestión y no solo un sistema contable.

Por su parte, (Toro, 2010) manifiesta que el costeo basado en actividades consiste en establecer costos a los elementos que se requieren para efectuar todas las tareas del proceso durante la producción, las mismas que son reconocidas como principales. Una actividad es una labor de consumo de recursos de una empresa y forma parte de un proceso que lo integran tareas con el fin de conseguir unas metas.

El ABC es identificado por las siglas en inglés Activity Based Costing y ha sido ampliamente conocido y puesto en uso en varias empresas con un nivel de tecnología avanzado, dejando obsoletos otros métodos tradicionales que se empleaban en los sectores productivos y fabriles ya que son notorias las ventajas que ofrece al momento de identificar y cuantificar los elementos que generan los costos directos e indirectos de manera exacta. Una vez encontrado el objeto del costo este sistema sigue los siguientes pasos: 
- Se estudia de forma ordenada el proceso productivo, identificando cada actividad que se ejecutó.

- Se analizan los costos y los volúmenes de recursos que se utilizaron.

- Se verifican los posibles elementos que generaron el costo de cada actividad, relacionando el motivo - desenlace y se establece una base considerando una fórmula de costos, cuyo valor se sustenta en la asignación unitaria.

- Se estudian los componentes de los costos por absorción por cada actividad para un determinado objeto de costo, posteriormente se elige la fórmula de absorción más indicada.

- Para calcular los costos del objeto, se suman los costos indirectos y lo directos. Se usa el costo así computando como base unitaria para situar costos a otros objetos y así abarcar todas las etapas de la fabricación.

(Lefcovich, 2007) señala algunos principios fundamentales para la aplicación el ABC/ABM, entre los más relevantes están los siguientes: gestionar la actividad y no el recurso que se va a gastar; utilizar los sistemas de costeo basado en actividades y presupuestos ABC como herramienta primordial en las organizaciones; ejecutar las actividades en función a los requerimientos y expectativas de los usuarios, y no de los objetivos internos de la empresa. Es decir, priorizar la importancia del cliente y no la de las autoridades; suprimir toda actividad que no esté generando valor alguno; crear sistemas en donde los productos y servicios cumplan con determinadas especificaciones, crear un ambiente ético donde la disciplina esté presente dentro de la organización, lo que ayudará a mejorar cada actividad o servicio que preste la empresa.

La gestión de costos por actividades al considerarse como una de las mejores herramientas que tienen a su favor las empresas de tipo industrial y en su búsqueda por lograr obtener un buen nivel de competencia en el mercado se proponen los siguientes objetivos: proveer de la información a todas las personas encargadas de la dirección de la empresa, esto se logrará gracias a los sistemas de información en cada área del negocio; agrupar todos los esfuerzos para acatar cada uno de los objetivos, con ayuda de los presupuestos; verificar las desviaciones y medidas correctivas y medir, 
analizar y presentar informes financieros y no financieros, todo esto conducirá a un desarrollo y posicionamiento competitivo de las organizaciones (López , 2011).

En este contexto, la gestión de costos se ha convertido en un aspecto primordial para las empresas, ya que tiene como objetivo básico el buscar soluciones a las limitaciones que se presentan en la información financiera ante aspectos importantes para la toma de decisiones. En el caso de las empresas industriales la toma de decisiones es complicada debido a que la contabilidad general y los sistemas de costeo tradicional no proporcionan la información suficiente y necesaria para la toma de decisiones. Lo mismo ocurre en el sector de la construcción donde los costos son importantes para medir y cuantificar de manera exacta las inversiones realizadas, su rentabilidad y utilidades, ante ello, se considera necesario generar una herramienta gerencial que permita proyectar tanto los costos como los ingresos de las obras o proyectos de construcción.

En el Ecuador, la construcción es considerada como uno de los sectores de gran productividad en la economía, las empresas constructoras dinamizan el sector económico del país, son generadoras de empleo, y proporcionan crecimiento y desarrollo económico a la nación. A este sector productivo de la economía, el gobierno lo vincula directamente como un factor significativo en el crecimiento del Producto Interno Bruto, el mismo que ha tenido un incremento acelerado debido a los préstamos bancarios otorgados al sector de la construcción por el Estado, esto ocasionó que la ciudadanía se incentive para tener su casa propia y en muchos casos comprar viviendas como un proyecto de inversión; por otra parte, el auge del precio del barril del petróleo durante los primeros años del siglo XXI hizo que el país invierta en construcciones de servicio social como puentes, carreteras, entre otros; sin embargo, en la actualidad la caída del precio del barril de petróleo y otros factores que han incidido en la economía nacional ha generado la disminución del porcentaje de construcción en el Ecuador, esta disminución ha causado pérdidas para todos los sectores económicos. Por estas razones, es imprescindible que las empresas constructoras; en tiempos de auge y recesión recurran a la incorporación de modelos de costos y presupuestación que permitan una integración de todo su circuito de gestión. 


\section{Metodología.}

El presente artículo tuvo un diseño no experimental, con un enfoque mixto donde se empleó herramientas cualitativas para elaborar las bases teóricas y cuantitativas para valorar los datos contables y estadísticos que se obtuvieron en el diagnóstico de la empresa OSMAR Constructores, el alcance de la investigación fue descriptivo - explicativo (DEXPLIS) para lo cual se hizo uso paralelo de los métodos inductivo y deductivo, e histórico - lógico; en relación al método inductivo - deductivo este par dialectico posibilitó la identificación de procesos y actividades en la empresa objeto de estudio; el histórico-lógico que permitió analizar las variables de estudio bajo un orden cronológico y con una secuencia lógica. En lo referente a las técnicas e instrumentos se utilizaron encuestas y entrevistas para obtener información a profundidad sobre las decisiones estratégicas de la empresa. El universo de estudio estuvo conformado por los empleados que trabajan en los departamentos financiero, contable, administrativo, así como también por los socios de la empresa que tienen relación directa con el manejo de la información contable - financiera.

\section{Resultados}

Como resultado de la aplicación de los instrumentos de investigación en la empresa OSMAR Constructores se determinó lo siguiente:

- No disponen de documentos, registros y procesos contables que les permitan conocer de forma adecuada los costos de producción.

- La constructora tiene identificadas de manera muy general todas las actividades principales, pero no posee una estructura formal y detallada de cada una de las actividades secundarias que intervienen en los procesos, ni las actividades que están organizadas en centros de costos.

- No determinar técnicamente la realización de sus presupuestos de obra, por lo que en ocasiones los precios calculados se encuentran por debajo del precio real o por encima del mismo, afectando su competitividad en el mercado.

- Se aplican controles a la ejecución de las obras permanentemente, sin embargo, no hay un control a la par de los procesos contables, en este sentido, existe una duplicidad de esfuerzos 
que contraponen criterios de las personas que intervienen en la toma de decisiones con quienes ejecutan los procesos contables.

- No disponen de una estructura contable que les permita contar con centros de costos, cuentas, subcuentas y control presupuestario.

- No disponen de una estructura organizacional sólidamente establecida, esto ha conllevado a que en varias circunstancias más de una persona realice la misma función o tome decisiones, provocando el incremento innecesario del costo de las obras.

- No siempre priorizan las necesidades relacionadas con las obras de construcción, por lo que se ha determinado un deficiente manejo de los rubros económicos obtenidos como anticipos, ya que estos fondos son utilizados para cubrir eventualidades y no siempre para financiar las actividades importantes de la construcción.

Bajo este escenario es relevante mencionar que el correcto manejo de la información genera a la gerencia una valiosa herramienta para la toma de decisiones, tanto al momento de determinar la política de precios, como al momento de generar un mix entre el costo y beneficio del producto y servicio. Por esta razón, se considera que el modelo de costeo por actividades determina de manera adecuada la carga de las actividades de apoyo y auxiliares, las mismas que afectan directamente al coste unitario del producto. Todo este proceso aparentemente no tendría un efecto final sobre el estado de resultados, sin embargo, al momento de realizar una correcta asignación de costes, se puede determinar a ciencia cierta si se generan utilidades o pérdidas por cada producto o servicio ofertado.

Por lo expuesto, se asume que la implementación de un modelo de costeo por actividades, permitirá aprovechar los recursos humanos, materiales y económicos dentro de la empresa OSMAR Constructores, lo que ayudará a obtener un mayor control en las operaciones y de esta forma se garantizará el logro de resultados positivos. De igual manera servirá como herramienta de apoyo para corregir cualquier desviación y obtener mejores resultados económicos. El modelo en su fase inicial parte con la identificación de los centros de costos y actividades para una obra de pavimentación, tal como se detalla a continuación: 


\section{Identificación de centros de costos y actividades.}

E modelo permite identificar cada uno de los procesos que intervienen en la ejecución de una obra, para luego distribuir de forma correcta cada actividad a los centros de costos y así poder determinar si estas se cumplen o no, como resultado se instauraran los costos que permiten ofrecer a los clientes un servicio óptimo y de calidad, así como también a los directivos contar con herramientas adecuadas para la toma de decisiones.

Figura 1. Identificación de centros de costeo y actividades.

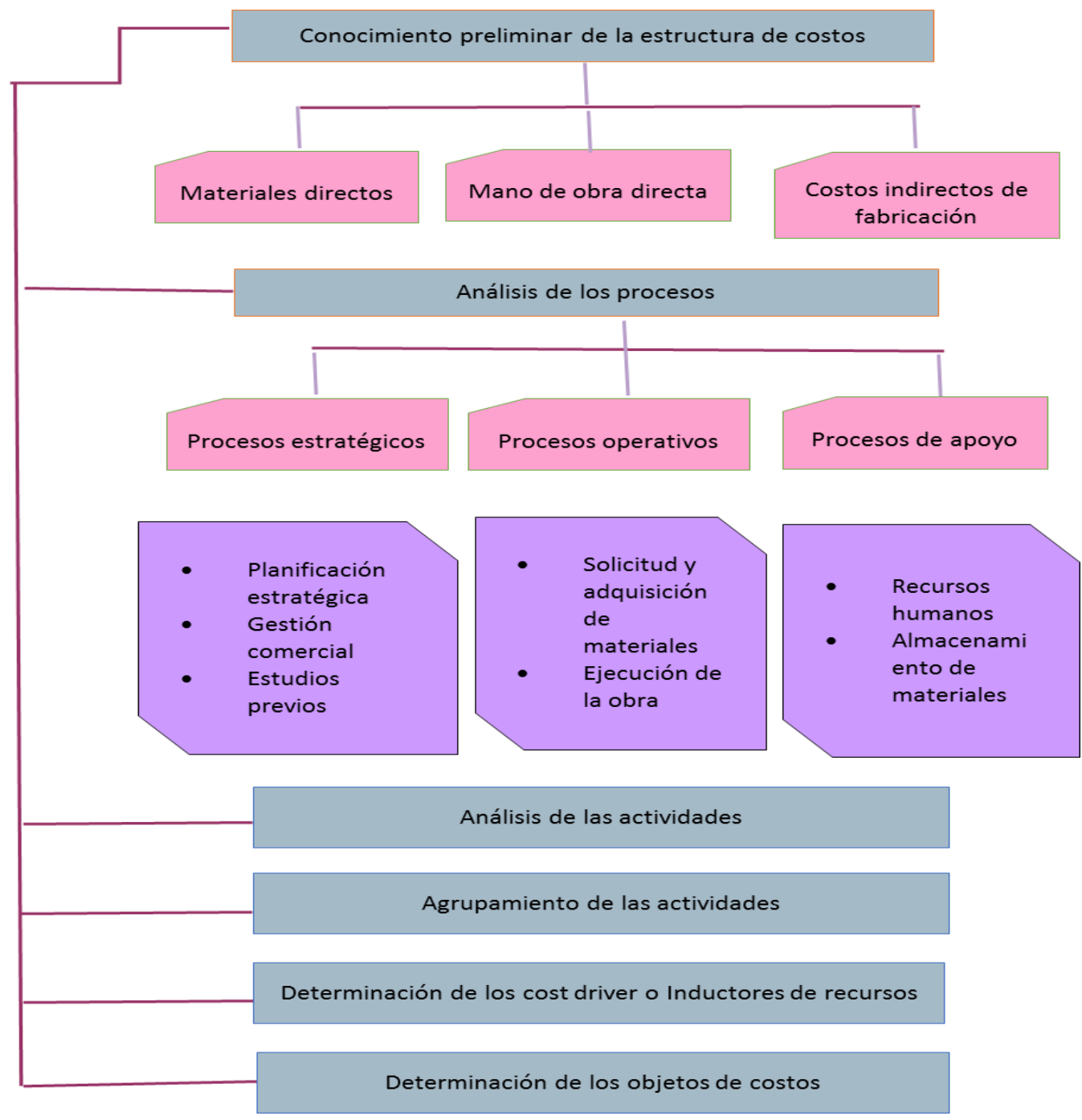


Considerando que una actividad es una acción efectuada ya sea por una persona o una máquina para la elaboración y entrega de algo a una tercera persona, es indispensable identificar cada una de las actividades que se presentan durante el proceso, por lo que es necesario conocer el área y al personal de trabajo, lo que facilitara la obtención de la información de recursos utilizados en las diferentes procesos, de igual forma, se determinara el tiempo de ejecución, y los subprocesos esenciales y las actividades que forman parte de las etapas presentadas en el esquema anterior. La empresa OSMAR constructores para la ejecución de las obras civiles deberá identificar la estructura de los costos que intervienen en la producción determinando de forma correcta la materia prima, mano de obra y los costos indirectos de fabricación.

Tabla 1. Costo directo e indirecto de la constructora OSMAR

\begin{tabular}{|l|l|}
\hline \multicolumn{1}{|c|}{ Costos directos } & \multicolumn{1}{c|}{ Costos indirectos } \\
\hline Volqueta & Combustible \\
\hline Tanquero & Lubricantes \\
\hline Rodillo ingersoli rand & Depreciación de maquinaria \\
\hline Mini rodillo ingersoli rand & Servicios básicos \\
\hline Motoniveladora caterpillar & Garaje \\
\hline Excavadora caterpillar & Aceites para la maquinaria y vehículos \\
\hline Excavadora de oruga komatsu & Ingeniero civil \\
\hline Retroexcavadora case & Arquitecto \\
\hline Retroexcavadora Caterpillar & Cuidadores de obra \\
\hline Minicargadora case & Operadores de maquinarias \\
\hline Minicargadora case & Operadores de maquinarias \\
\hline
\end{tabular}

Materiales Directos; - La adquisición de los materiales que se utilizaran dentro del proceso de ejecución de una obra, dependerá de las especificaciones de los clientes y de la orden de trabajo que se realizara para el control del avance del proceso. 
Mano de obra directa. - Es importante que se lleve un control adecuado de la mano de obra y así asignar un costo real a la producción.

Costos indirectos de fabricación. - Dentro de estos costos se encuentran los materiales indirectos, mano de obra indirecta y otros costos indirectos de fabricación que están conformados por aquellos valores que intervienen de forma indirecta dentro del proceso de producción.

Tabla 2. Clasificación del costeo indirecto

\begin{tabular}{|l|}
\hline \multicolumn{1}{|c|}{ Materia prima indirecta } \\
\hline Combustible \\
Lubricantes \\
\hline Aceites para vehiculo \\
\hline \multicolumn{1}{|c|}{ Mano de obra indirecta } \\
\hline Ingeniero civil \\
\hline Arquitecto \\
\hline Cuidador de obra \\
\hline Cuidador de maquinaria \\
\hline \multicolumn{1}{|c|}{ Costos indirectos de fabricación } \\
\hline Depreciaciones de maquinaria \\
\hline Servicios basicos \\
\hline
\end{tabular}

\section{Análisis de los procesos}

El sistema de costeo ABC diseña un mapa de las actividades que se desarrollan durante el proceso de ejecución de la obra. Esta etapa resulta una herramienta muy útil al momento de mejorar los procesos, al conocer el tiempo, la cantidad y costo que consumen cada actividad.

Dentro de esta etapa se pretende identificar y clasificar cada una de las actividades, como primarias o de apoyo, resaltando la adecuada clasificación de los diferentes procesos que son considerados como de apoyo, los mismos que generan un valor significativo al finalizar la ejecución de la obra. Dentro de las empresas constructoras resulta complicado identificar cada uno de los pasos que se sigue en el proceso de ejecución de la obra, muchas de las operaciones son principales y existen otras implícitas que generan costos ocultos. 


\section{Procesos estratégicos}

Planificación estratégica: En la actualidad la constructora no cuenta con una misión, visión y un organigrama estructural que le permitan conocer de forma adecuada la estructura jerárquica, lo que le ha impedido distribuir de manera adecuada las funciones al personal que se encuentran laborando dentro de la constructora. Con la finalidad de llevar a cabo la aplicación del modelo se propone una estructura organización y el planteamiento estratégico para la empresa.

Figura 2. Estructura organizacional.

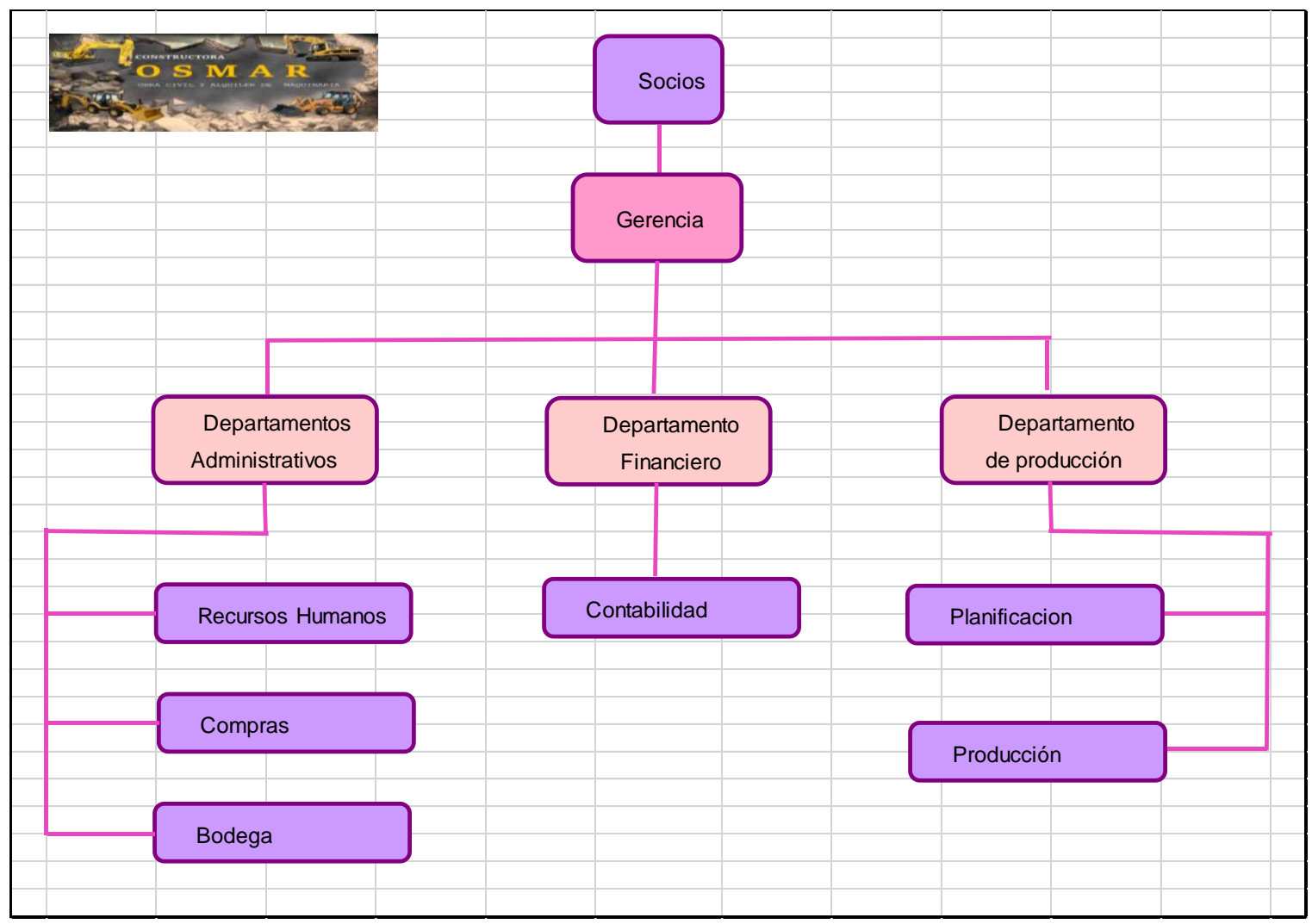

\section{Misión.}

OSMAR Constructores es una empresa especializada y con años de experiencia en brindar los servicios de asesoría en construcciones civiles, mantenimiento y construcción de carreteras, vías, puentes, etc. Estamos integrados por profesionales idóneos y altamente capacitados, mano de obra calificada y maquinaria adecuada para desarrollar los diferentes proyectos de construcción, y poder 
cumplir con nuestra meta principal que es el de satisfacer todas las exigencias de nuestros clientes, desarrollando nuestro trabajo con un alto grado de responsabilidad y cumplimiento, ofreciendo servicio de calidad.

Visión.

Consolidar nuestro liderazgo y reconocimiento en el sector de la construcción, en términos de productividad y especialización para la ejecución de obras civiles, de manera que se fortalezca el crecimiento económico - financiero de la constructora, manteniendo siempre los más altos estándares de calidad.

Gestión comercial: este paso es considerado como la herramienta que permite entrar al mercado buscando clientes y presentado ofertas dentro del portal de compras públicas para obras civiles en el sector público, es decir, permite que la constructora ingrese al ambiente de competencia.

Estudios previos: lo conforman todos los estudios técnicos previos a la elaboración de la obra civil, aquí se revisarán todos los documentos solicitados por la parte contratante.

\section{$\underline{\text { Procesos Operativos }}$}

Solicitud y adquisición de materiales: este paso se constituye en el primer acercamiento con el cliente con la finalidad de conocer el tipo de obra a ejecutarse, y poder realizar el detalle del tipo de producto, especificaciones, utilización, modelo, etc.

Ejecución de obra: una vez realizado el primer anticipo por la parte contratante se procede a realizar la orden de trabajo, estableciendo en ella la distribución del personal, horas de trabajo, tareas a desarrollar y control del consumo de materiales.

Procesos de apoyo

Recursos Humanos: una vez que se cuenta con los materiales el siguiente paso es buscar el recurso humano necesario que cuente con la experiencia necesaria en ejecución de obras civiles.

Almacenamiento de materiales: Los materiales adquiridos son llevados al lugar donde se ejecutará la obra y son recibidos por el bodeguero y capataz quienes se encargan de verificar si todos los materiales cumplen con los requerimientos previamente solicitados. 


\section{Análisis de las actividades y agrupación de las actividades}

Esta etapa es el punto central del sistema de costos ABC/ABM, y es la etapa en la cual se determina las actividades que se desarrollan en cada uno de los centros de costos.

Para que un sistema de costos cumpla de manera satisfactoria su función dentro de una empresa es necesario que esta tenga una buena organización. Para lo cual resulta de gran importancia que todos los factores de la producción se encuentren coordinados. Por lo que un sistema de costos de calidad deberá diseñarse en coordinación con el sistema de control de la producción.

Tabla 3. Costos directos e indirectos de la Constructora OSMAR

Método ABC

Estado de resultados (4 meses de ejecución)

\begin{tabular}{lr|lr}
\hline \multicolumn{1}{c}{ Costos directos: } & Cos to total & Costos indirectos : & Costo total \\
Materiales & $204.775,00$ & Servicios básicos & 730,00 \\
Mano de obra & $59.200,00$ & Sueldos y salarios & $9.481,28$ \\
Depreciación maquinaria & $19.833,33$ & & \\
Combustibles & $7.250,00$ & & \\
Lubricantes & $1.500,00$ & & $10.211,28$ \\
& & & \\
Total directo & $292.558,33$ & Total & \\
Total costos & $302.769,61$ & &
\end{tabular}

Tabla 4. Estado de resultados tradicional

Método tradicional

Estado de resultados (4 meses de ejecucion)

\begin{tabular}{lr|lr}
\hline Costos directos: & Costo total & \multicolumn{1}{c}{ Costos indirectos : } & Cos to total \\
Materiales & $204.775,00$ & Servicios básicos & 730,00 \\
Mano de obra & $59.200,00$ & Sueldos y salarios & $9.481,28$ \\
& & Depreciación maquinaria & $19.833,33$ \\
& & Combustibles & $7.250,00$ \\
& & Lubricantes & $1.500,00$ \\
\hline Total directo & $263.975,00$ & Total & $38.794,61$ \\
Total costos & $302.769,61$ & &
\end{tabular}

Al determinar costos directos e indirectos por el método tradicional, en muchas ocasiones se confunden la clasificación y terminan clasificándose costos variables por fijos, como es el ejemplo de la depreciación que, en este caso, está determinada en función directa de su uso o trabajo 
Tabla 5. Identificación de costos y actividades en OSMAR constructores.

\begin{tabular}{|c|c|c|c|}
\hline Centro de costos & Actividades & Inductores & Responsable \\
\hline \multirow[t]{8}{*}{$\begin{array}{l}\text { Postulación de } \\
\text { ofertas }\end{array}$} & Recepción de pedido o postulación en concurso & $\begin{array}{l}\text { Número de } \\
\text { proyectos }\end{array}$ & Gerente \\
\hline & $\begin{array}{l}\text { Preparación de información de acuerdo a las } \\
\text { especificaciones de los solicitado por el cliente. }\end{array}$ & $\begin{array}{l}\text { Número de } \\
\text { proyectos }\end{array}$ & Gerente \\
\hline & Elaboración de la proforma. & $\begin{array}{l}\text { Número de } \\
\text { proyectos }\end{array}$ & Administrador \\
\hline & Revisión y aprobación de la proforma. & $\begin{array}{l}\text { Número de } \\
\text { proyectos }\end{array}$ & Gerente \\
\hline & Revisión y firma del contrato. & $\begin{array}{l}\text { Número de } \\
\text { proyectos }\end{array}$ & Gerente \\
\hline & Ingreso de la orden de trabajo. & $\begin{array}{l}\text { Número de } \\
\text { proyectos }\end{array}$ & Administrador \\
\hline & Ingreso del anticipo & $\begin{array}{l}\text { Número de } \\
\text { proyectos }\end{array}$ & Administrador \\
\hline & Archivo y entrega de la documentación generada & $\begin{array}{l}\text { Número de } \\
\text { proyectos }\end{array}$ & Administrador \\
\hline \multirow[t]{5}{*}{ Pavimentación } & Replanteo y nivelacion de eje & $\begin{array}{l}\text { Horas hombre / } \\
\text { mts } 2\end{array}$ & Operador \\
\hline & Excavacion sin clasificar maquinaria & $\begin{array}{l}\text { Horas hombre / } \\
\text { mts } 2\end{array}$ & Operador \\
\hline & Excavacion de estructuras menores & $\begin{array}{l}\text { Horas hombre / } \\
\text { mts } 2\end{array}$ & Operador \\
\hline & Cargada de material con maquinaria & $\begin{array}{l}\text { Horas hombre / } \\
\text { mts } 2\end{array}$ & Operador \\
\hline & Acabado de la obra basica & $\begin{array}{l}\text { Horas hombre / } \\
\text { mts } 2\end{array}$ & Operador \\
\hline \multirow[t]{8}{*}{$\begin{array}{l}\text { Red de distribución } \\
\text { de agua potable }\end{array}$} & $\begin{array}{l}\text { Excavacion de zanja a maquina en material sin } \\
\text { clasificar }\end{array}$ & $\begin{array}{l}\text { Horas hombre / } \\
\text { mts } 2\end{array}$ & Operador \\
\hline & Excavacion de estructuras menores & $\begin{array}{l}\text { Horas hombre / } \\
\text { mts } 2\end{array}$ & Operador \\
\hline & Relleno compacto con compactador mecanico & $\begin{array}{l}\text { Horas hombre / } \\
\text { mts } 2\end{array}$ & Operador \\
\hline & Anclaje de hormigon simple & $\begin{array}{l}\text { Horas hombre / } \\
\text { mts } 2\end{array}$ & Operador \\
\hline & Cargada de material con maquinaria & $\begin{array}{l}\text { Horas hombre / } \\
\text { mts } 2\end{array}$ & Operador \\
\hline & Transporte de material & $\begin{array}{l}\text { Horas hombre / } \\
\text { mts } 2\end{array}$ & Operador \\
\hline & Pruebas hidrostaticas & $\begin{array}{l}\text { Horas hombre / } \\
\text { mts } 2\end{array}$ & Operador \\
\hline & Desinfeccion de tuberias & $\begin{array}{l}\text { Horas hombre / } \\
\text { mts } 2\end{array}$ & Operador \\
\hline
\end{tabular}


El segundo paso en la adaptación del sistema de costeo ABC es identificar los centros de costos y sus actividades.

Tabla 6. Distribución de costos por obra

\begin{tabular}{lllll} 
& Obra 1 & Obra 2 & Obra 3 & Total \\
\hline Materiales & $50.152,00$ & $71.592,00$ & $83.031,00$ & $204.775,00$ \\
Mano de obra & $17.266,67$ & $19.733,33$ & $22.200,00$ & $59.200,00$ \\
CIF & $11.315,10$ & $12.931,54$ & $14.547,98$ & $38.794,61$ \\
\hline Costo total & $\mathbf{7 8 . 7 3 3 , 7 6}$ & $\mathbf{1 0 4 . 2 5 6 , 8 7}$ & $\mathbf{1 1 9 . 7 7 8 , 9 8}$ & $\mathbf{3 0 2 . 7 6 9 , 6 1}$ \\
Unidades mt2 & 350,00 & 400,00 & 450,00 & $1.200,00$ \\
\hline Unitario & 224,95 & 260,64 & 266,18 &
\end{tabular}

Se presenta el costeo por obra ejecutada en un estado de resultado segregado

Tabla 7. Distribución de costos por centros.

\begin{tabular}{lrrrr}
\hline & \multicolumn{3}{c}{ Centro de costos } & \multicolumn{3}{c}{$\begin{array}{c}\text { Red de } \\
\text { distribución } \\
\text { Costos directos: }\end{array}$} & $\begin{array}{c}\text { Postulación } \\
\text { de ofertas }\end{array}$ & Pavimentación & Total \\
potable & \\
\hline Materiales & & $153.581,25$ & $51.193,75$ & $204.775,00$ \\
Mano de obra & $44.400,00$ & $14.800,00$ & $59.200,00$ \\
$\begin{array}{l}\text { Depreciación } \\
\text { maquinaria }\end{array}$ & $14.875,00$ & $4.958,33$ & $19.833,33$ \\
Combustibles & & $5.437,50$ & $1.812,50$ & $7.250,00$ \\
Lubricantes & $1.125,00$ & 375,00 & $1.500,00$ \\
Servicios básicos & 730,00 & & & 730,00 \\
Sueldos y & & & & $9.481,28$ \\
salarios & $9.481,28$ & $\mathbf{2 1 9 . 4 1 8 , 7 5}$ & $\mathbf{7 3 . 1 3 9 , 5 8}$ & $\mathbf{3 0 2 . 7 6 9 , 6 1}$ \\
Total & $\mathbf{1 0 . 2 1 1 , 2 8}$ & $\mathbf{2 1 0}$ &
\end{tabular}

La tabla 7 muestra la distribución de los costos por centros de costos.

Como se observa en la tabla 6 y la tabla 7 los costos totales para el periodo de ejecución de las 3 obras de construcción son de $\$ 302.769,61$, que permanece invariable tanto para la clasificación por costeo tradicional en la tabla 3, como al final del proceso. 


\section{Conclusiones}

- Toda empresa de construcción tiene como principal objetivo la maximización de sus resultados financieros, medirlos resulta objeto de estudio de una gran cantidad de investigaciones a nivel regional y nacional, por lo que se ha establecido que los métodos tradicionales no logran satisfacer de manera eficiente las necesidades de este sector, en este sentido, el modelo de costos $\mathrm{ABC}$ aparece como una alternativa moderna que permite contar con información más real en cuanto a la determinación de los costos, precios y presupuestos de las obras y proyectos de construcción.

- Diseñar un modelo de costeo ABC es una tarea compleja en el sector de la construcción, ya que, a más de conocer las características particulares y requerimientos de las empresas, es necesario identificar a profundidad las operaciones relacionadas con el sistema de costeo, para establecer las actividades que conviene mantener en la empresa y las que se deben eliminar, a partir de ello, el modelo permitirá la determinación de los costos con una distribución más exacta de los costos indirectos.

- La principal causa para implantar un modelo de contabilidad de costeo por actividades en la empresa OSMAR constructores es la falta de información que permita aprovechar los beneficios que esta brinda. Identificando adecuadamente cada uno de los centros de costos, así como las actividades que intervienen en cada uno de ellos.

- La implementación del modelo de costeo basado en actividades por parte de la administración de la empresa objeto de estudio, permitirá la adecuada toma de decisiones en el ámbito económico y contable, ya que la empresa contará con datos reales, además, de una adecuada estructura de costes que propiciará la optimización de recursos y corregirá todas las actividades que restan valor y generan gastos excesivos en los centros de costos de la constructora, encareciendo el producto y servicio final. 


\section{Referencias Bibliográficas}

Amat y Soldevila. (2011). Contabilildad y Gestión de Costos. Barcelona : Profit Editorial.

Calvo, M. (27 de Diciembre de 2017). Contabilidad de gestión. Obtenido de Captio: https://www.captio.net/blog/contabilidad-de-gestion-en-las-pymes

Chezpik Garcia, A. (16 de Mayo de 2007). Contabilidad de gestión y de Costos. Obtenido de Gestiopolis: https:/www.gestiopolis.com/contabilidad-de-costos

Estupiñan, R. (2006). Analisís Financiero y de Gestión. Bogota: Ecoe Ediciones.

Jiménez Lemus, W. (2010). JContabilidad de Costos. Bogotá: Funcacion para la Educación Superior San Mateo.

Kaplan y Cooper . (2006). Costos y efecto. Madrid: Ediciones Gestión.

Lawrence, W. (2002). Contabilidad de costos. Monterrey: Montaner y Simon S.A Editores.

Lefcovich, M. (18 de Diciembre de 2007). Gestión basada en actividades ABM. Obtenido de Gestiopolis: https//www.gestiopolils.com/gestión-basado-en-actividades-abm

López , C. (2011). Sistema de costos. México: Editorial Prentice Hall.

Lopéz Rodriguez, M. (2011). La contabilidad de gestión, evolución y particularidades. Ilustrados. Molina, A. (2002). Contabilidad de costos. Quito: Nickerson Editores.

Toro, F. (2010). Costos ABC y presupuesstos herramientas poara la productividad. Colombia: Ecoe Ediciones.

Valdez, Nazareno y Toala. (2017). Una visión genenal acerca de la contabilidad de gestión . Revista cientifíca Dominio de las ciencias.

Zapata Sanchéz, P. (2007). Contabilidad de costos herramienta para la toma de decisiones. Bogotá: Editorial Mc Graw - Hill Interamericana S.A.

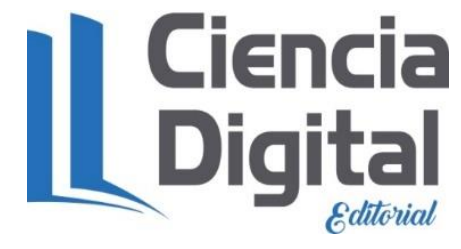




\section{PARA CITAR EL ARTÍCULO INDEXADO.}

Escandón Guillén, P., Narváez Zurita, C., Eràzo Álvarez, J., \& Ormaza Andrade, J. (2019). La gestión de costos basada en actividades como herramienta estratégica para la toma de decisiones en las empresas constructoras de la ciudad de Loja, Ecuador. Visionario Digital, 3(2.1.), 368-387. https://doi.org/10.33262/visionariodigital.v3i2.1.581

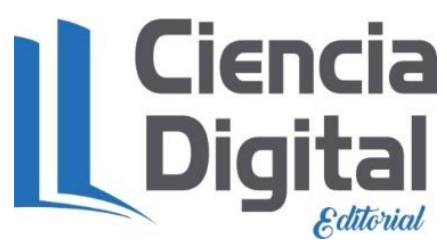

El artículo que se publica es de exclusiva responsabilidad de los autores y no necesariamente reflejan el pensamiento de la Revista Ciencia Digital.

El artículo queda en propiedad de la revista y, por tanto, su publicación parcial y/o total en otro medio tiene que ser autorizado por el director de la Revista Ciencia Digital.
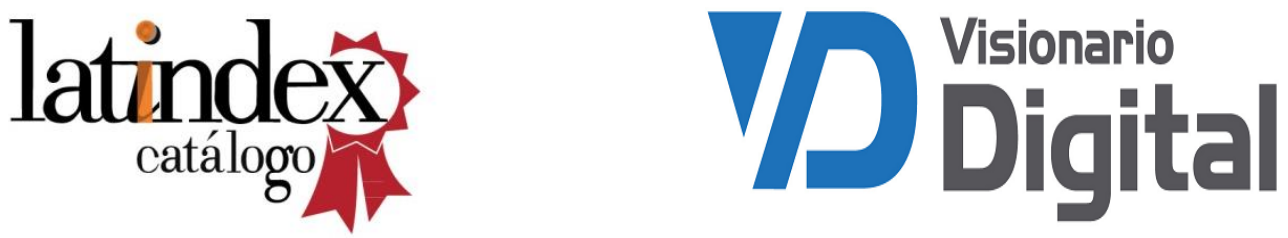